Covered in: Web of Sciences (WOS); EBSCO; ERIH+; Google Scholar; Index Copernicus; Ideas RePeC; Econpapers; Socionet; CEEOL; Ulrich ProQuest; Cabell, Journalseek; Scipio; Philpapers; SHERPA/RoMEO repositories; KVK; WorldCat; CrossRef; CrossCheck

2019, Volume 11, Issue 1, pages: 177-186 | doi: https://doi.org/10.18662/rrem/104

\section{Attitude and Self- Efficacy of Students with Motor Impairments Regarding Touch Input Technology}

\section{Maria-Doina SCHIPOR ${ }^{1}$}

${ }^{1}$ Associated professor, "Ștefan cel Mare" University, Suceava, Romania, doina.schipor@usm.ro
Abstract: We address in this work the role touch interfaces could play in reducing the inequalities motor disabled students confront with. To this end, we conduct a between subjects experiment, involving students with motor disability and able bodied participants, investigating both the attitude and the selfefficacy with respect to the utilisation of touch technology in the everyday life. We rely on the well-established relationship between attitude, self-efficacy, and actual performance in order to explore strategies for increasing the actual performances through psychological means. The statistical analysis indicates significant differences between subjects with motor impairments and able bodied participants in the case of both attitude and selfefficacy. We connect these outcomes with strategies for reducing the inequalities for the students with motor disabilities in the context of educational activities.

Keywords: Motor disabilities; touch technology; attitude; self-efficacy; inclusive design.

How to cite: Schipor, M.-D. (2019). Attitude and SelfEfficacy of Students with Motor Impairments Regarding Touch Input Technology. Revista Romaneasca pentru Educatie Multidimensionala, 11(1), 177-186. https://doi.org/10.18662/rrem/104 


\section{Introduction}

People with motor impairments have to access IT\&C systems in order to gain a better control over their bodies and surrounding environment and to extend their social involvement. Touchscreens become the "de facto" interface for all smart devices that ubiquitous coexist all around us. That is why, understanding the way mobility disabled people use touch technology is a key step in helping them to live a closer to normal life. The main majority of related studies focuses on the increasing touch input performance through technical means (i.e. software and hardware improvements). However, although often neglected, some psychological aspects of interaction (Schipor \& al., 2011) such as attitude and selfefficacy could also lead to an increasing in performance by offering the motivational background for learning and adaptation to this new technology. In this paper we investigate the attitude and the self-efficacy of subjects with motor impairments and able-bodied people in relation with touchscreens.

\section{Literature Review}

\subsection{Motor Disabled People and Touchscreens}

Motor disabilities are physical impairments related with deficiencies in movement, coordination, and sensation (Tai, Blain \& Chau, 2008). Similarly with other types of disabilities (Schipor \& Vatavu, 2017; Schipor \& Schipor, 2007), the main majority of these impairments drastically reduces the ability to perform common activities, and to interact with others (WHO, 2011). Although the classical stereotypical view associates motor impairments with wheelchairs, the reality is far more complex. Thus, while the percent of people diagnosed as motor disabled is around 6.5\% (Kraus, 2017) it must be noted that the majority of people experience some form of temporal or permanent motor deficiencies during their lives (WHO, 2011).

IT\&C is often viewed as a way to reduce the gap between ablebodied and people with motor impairment helping the latter to extend their control range. In the last two decades the IT\&C term extends its meaning to include not only the classical PCs but also all ubiquitous smart devices such as tablets, smartphones, wearable gadgets, etc. The technology acts as an interface between person and physical surrounding environment (e.g. smart homes) but also as a social connector with other people (e.g. social media) (Tai, Blain \& Chau, 2008). 

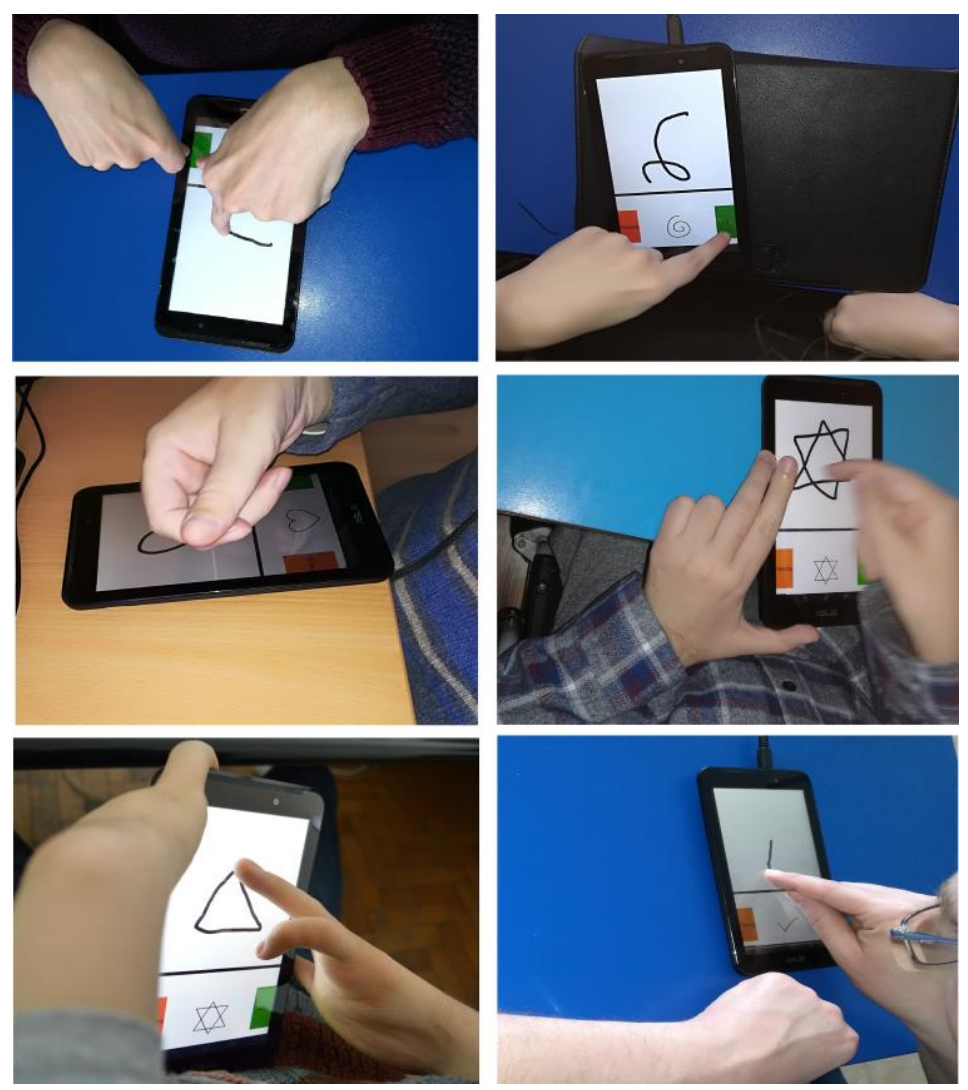

Figure 1. Six of the students with motor disabilities involved in our study working with touchscreens. Five mobility impairments are highlighted: spastic tetraparesis (top), spinal cord injury tetraplegia (middle-left), muscular atrophy (middle-right), phocomelia (bottom-left), and muscular dystrophy

(bottom-right).

Providing an easy interaction with computers is in the very short list of the most important integrative techniques for the people with motor disabilities. Several alternative interfaces have been invented and tested during la last decades, including touchscreens, eye takers, voice input, wearable devices or electroencephalography (EEG) headsets in order to surpass the classical mouse and keyboard (Tai, Blain \& Chau, 2008). Nevertheless, none of these technologies work in the same way for all of the motor disabilities because the performance and usability greatly vary with the type and the level of impairment. The touchscreens become the most wellknown and widespread alternative interface due to the impressive increase of 
smart mobile devices utilization. This evolution goes hand in hand with the miniaturization and the spreading of computing power into tiny devices, unsuitable for dedicated mouse and keyboard (e.g. smartphones, smartwatches, ATMs, controlling interfaces). However, experiments trying to understand how people with motor disabilities deal with these interfaces, remain relatively sparse (Gorce \& al., 2017). We believe that addressing the issues of attitude and subjective perception on self-efficacy is a key step in formulating recommendations for increasing the touch input performance.

\subsection{Attitude and Self-efficacy}

Attitude is a central concept of social psychology. Although the exact definition evolved over the years (Schwarz \& Bohner, 2001), the main majority of scientific community agrees that an attitude reflects the subjective tendency to like or dislike a particular entity (Eagly \& Chaiken, 1993, 2007). Many models have been proposed in order to describe the inner intricate structure of attitudes (Jain, 2014). The earlier models such as Expectancy-Value Model (Calder \& Lutz, 1972), Multi-attribute Measurement Model (Fishbein, 1963), and Vector Model (Calder \& Lutz, 1972) highlight the affective and cognitive basis of attitudes. However, more recent approaches such as Tripartite Model and ABC Model (Eagly \& Chaiken, 1993) put forward a third component, the behavior. In this paper we rely on the following components of attitude (Jain, 2014; Breckler, 1994): Affective - feelings or emotions about the entity, Behavioral - actions related with the entity, and Cognitive - beliefs and thoughts regarding the entity.

In his seminal paper, Bandura (1982) defines self-efficacy as a subjective perception about "how well one can execute courses of action required to deal with prospective situations". There are two important findings regarding self-efficacy: a) it is connected with attitude and b) it influences the actual efficacy (Schwarzer \& Luszczynska, 2005). Thus, by inducing a positive tendency about a task (attitude) it is possible to increase the confidence in successfully achieving that task (self-efficacy) and to actually obtain more power to face the challenges related with that goal. That is why it is very important to understand not only the technical parameters (e.g. screen size, touch surface sensitivity, etc.) but also the psychological mechanisms that could lead to an enhanced touch input performance.

\subsection{Comparative studies}

Relatively few studies have been published on understanding the rules that govern the interaction of people with motor impairments with 
touchscreens when compared with able-bodied (Gorce \& al., 2017; Findlater $\&$ al. 2017). Moreover, the attitudinal aspects were often neglected because the experiments were mostly performed by the technical researchers without the participation of psychology specialists. The closest results refer to other categories of people experiencing difficulties in using touch interfaces such as children (Vatavu \& al., 2015), visual impaired (Schipor \& Vatavu, 2017), and elderly. Gorce et al. (2013) reviewed thirty six research papers that evaluated interaction techniques in the case of elderly using touchscreen devices. This study identified several parameters that influence touchscreen interaction such as users' skills, previous experience, cognitive capabilities, and attitude. It also highlighted the limitations of previous experiments including the lack of standardization and investigator and devices impact.

In his work of 2017, Page analyzes the differences in attitude and perceived usability between younger (18-25 age range) and older (60-70 age range) people. He found out that there are differences in the case of interaction styles (e.g. two hands - younger, one hand - older), preferred apps (e.g. Twitter and Facebook - younger, Calendar and Camera - older), brand (e.g. iPhone - younger, not important - older), and preferred interaction type (e.g. touch - younger, push buttons - older). However, the attitude regarding touch technology seems to be positive or negative mainly due to the previous experiences, age being only a contributing factor. Another pilot study (Chiu \& al., 2016) highlights the impact of utilization of touchscreen devices in changing attitude toward touch technology. Regular utilization of touchscreen smartphones has the potential to improve psychical health and to reduce the reluctance towards utilization of new technologies. As presented above, the closest related studies addressing the attitude and self-efficacy in using the touch interfaces involved elderly people. Although both older and people with motor disabilities share some difficulties in using touchscreens, we consider it is wrong to simply extend the conclusions drawn from these experiments to another category of users. Therefore, the main contribution of this paper is to investigate the specific attitude and self-efficacy of people with motor impairments in relation with touch interfaces.

\section{Experiment}

We employed a between subject experiment to understand the effect of motor disability on attitude and self-efficiency with respect to touch devices. 
Attitude and Self-Efficacy of Students with Motor Impairments Regarding ...

\subsection{Participants, apparatus, and task}

Eighteen volunteered students (age $\mathrm{M}=26.33, \mathrm{SD}=6.71$ years, 5 female) were involved in our experiment. This study design was approved by the Research ethics committee of the Stefan cel Mare University of Suceava (protocol number 16854/26.10.2017). The study was conducted according to the declaration of Helsinki principles. A specific and detailed written informed consent was obtained for all participants in our data collection experiment. We employed one independent variable - SUBJECT-CONDITION - with two categories: able-bodied ( 9 subjects) and motor impaired (9 subjects). The participants are affected by the motor disabilities resulting in partial or total loss of use of hands (Figure 1): spastic tetraparesis (3), spinal cord injury tetraplegia (2), muscular dystrophy (2), phocomelia (1), and spinal muscular atrophy (1). The subjects received a questionnaire consisting in 14 questions from which 8 addressed self-efficacy and 6 addressed attitude. The participants full-filled the responses in 5 points and 4 points Likert scales, the former for the attitude related items and the latter for the self-efficiency items. All the subjects have had previous experience in using touch devices (smartphones or tablets). Although no time restrictions were imposed, no one spent more than five minutes on these items.

\subsection{Measures}

The responses were averaged in order to obtain the following factors (quantitative dependent variables):

- AtTitude-AfFective - what the participants feel (pleasure, satisfaction) about using touchscreens;

- AtTitude-BEHAVIOR - how the subjects actually use (frequency, experience) touch devices;

- Attitude-Cognitive - what the participants think (easiness of use) about touch interaction;

- SELF-EFFICACY - what subjects think about their efficiency in accomplishing a task using touch technology.

For all the attitude related variables, the score ranges between 1 (indicating a negative attitude toward touchscreens) and 5 (showing a positive attitude). The SELF-EFFICACY variable takes values between 1 (denoting a low perceived self-efficacy in using touch devices) and 4 (indicating a high perceived self-efficacy in tasks involving touchscreens). 


\section{Results and Discussions}

Figure 2 shows the average values for all dependent variables for each category of the SUBJECT-CONDITION variable. All attitude related dependent variables (i.e. AtTITUde-AfFEctive, AtTITUDE-BEHAVIOR, and AtTITUDE-Cognitive) are grouped in the first graph while the SELFEFFICACY is represented in the second graph. Due to differences in Likert scales, the maximum score value is 5 for the first three variables and 4 for the forth variable. The red lines between groups indicate statistical significant differences related with the corresponding dependent variables.

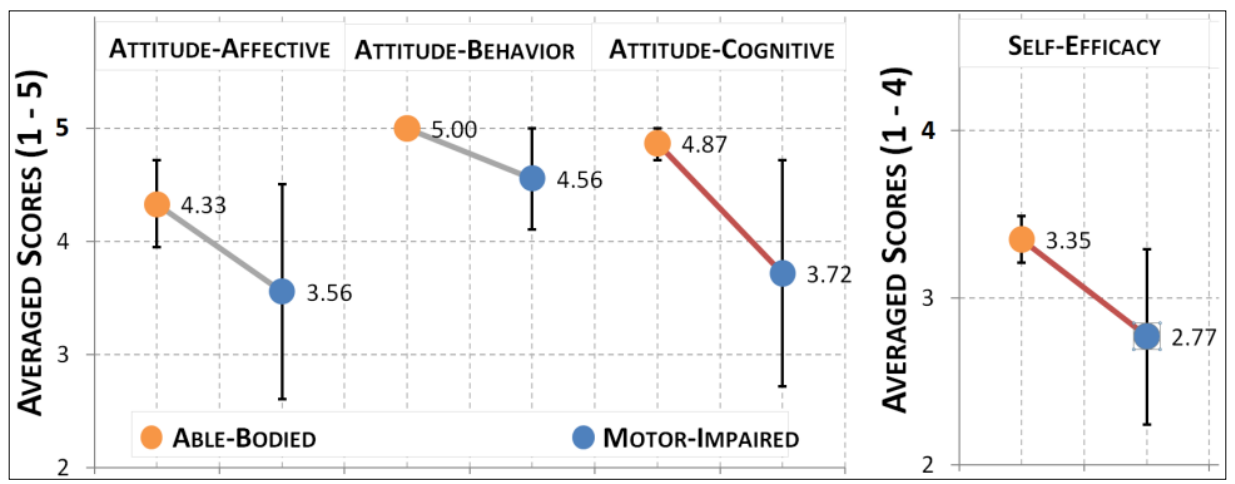

Figure 2. Averaged scores for all dependent variables, for each category of the SUBJECT-CONDITION variable. Errors bars show 95\% confidence intervals for means. Red lines indicate a statistical significant difference between groups.

In the case of all variables, the group of students with motor impairments obtained lower averaged values when compared with its ablebodied counterpart. A Mann-Whitney test indicated that the AttitudeCognitive was significantly greater for able-bodied participants (Median = 5.00, Mean rank $=12.17$ ) than for their motor impaired counterparts (Median $=4.00$, Mean rank $=6.83), \mathrm{U}=16.50(\mathrm{Z}=-2.33), \mathrm{p}=.031$, with a large size effect $(r=.549)$. Similarly, the students without motor disabilities had a marginally significant higher Self-Efficacy score (Median $=3.38$, Mean rank $=12.00)$ that the others participants (Median $=3.00$, Mean rank $=$ 7.00), $\mathrm{U}=18.00(\mathrm{Z}=-2.01), \mathrm{p}=.050$ with a medium to large size effect $(\mathrm{r}$ $=.474)$. However, no significant differences were detected between the two groups in the case of the Attitude-Affective $(\mathrm{U}=26.50, \mathrm{Z}=-1.28, \mathrm{p}=.222)$ and in the case of the Attitude-Behavior $(\mathrm{U}=27.00, \mathrm{Z}=-1.84, \mathrm{p}=.257)$.

A Kendall's tau-b correlation was run to determine the relationship between each attitude related variables and the Self-Efficacy, for all the 
participants. There was a strong, positive correlation between Self-Efficacy and both Attitude-Affective (Tau-b = .655, $\mathrm{p}=.001)$ and Attitude-Cognitive $($ Tau-b $=.706, \mathrm{p}<.001)$. However, there was no correlation between SelfEfficacy and Attitude- Behavior (Tau-b $=.298, \mathrm{p}=.152)$.

Although the two groups of participants feel and use touch devices in a similar way, they differ in terms of cognitive dimension of attitude. Thus, the subjects with motor impairments evaluate touch tasks as being more challenging. These results indicate that despite their impairment, the subjects consider touch devices appropriate and desirable for their daily activities but, however, they cannot deny the encountered difficulties. These difficulties are also expressed by the self-efficacy scores indicating a lower self-confidence. The correlation between self-efficacy scores and affective and cognitive dimensions confirms the connections highlighted by previous studies. In the context of education, using touch screen devices for students with motor impairments can determine lower performances and lower selfefficacy levels. However, due to the positive affective and behavioural attitude towards touchscreens, the limitation of touch technology utilisation can also be a source of inequalities. That is why we recommend not to limit access to these educational instruments but to adopt supporting strategies such as increasing time for tasks and adapting software apps.

\section{Conclusion}

In this paper we presented a study investigating the attitude and the self-efficacy of people with motor disabilities in relation with touch technology utilization. We compared this group of participants with its ablebodied counterpart in order to make these results useful in the context of reducing inequalities. We found the groups similar in terms of both affective and behavioral components of attitude and different in terms of self-efficacy and cognitive component of attitude. These outcomes could help the research community to formulate strategies for encouraging people with disabilities to become more self-confident in using touchscreens as a psychological way for increasing touch input performance. We also hope that this exploration will foster further research in order to validate and enhance our approach.

\section{Acknowledgement}

This work was supported by a grant of the Romanian National Authority for Scientific Research and Innovation, CNCS/CCCDI - UEFISCDI, project number PN-III-P2-2.1-PED-2016-0688 (209PED/2017) within PNCDI III. 
The study was approved by the Research ethics committee of the Stefan cel Mare University of Suceava (protocol number 16854/26.10.2017).

\section{References}

Bandura, A. (1982). Self-efficacy mechanism in human agency. American psychologist, $37(2), 122$.

Breckler, S. J. (1984). Empirical validation of affect, behavior, and cognition as distinct components of attitude. Journal of personality and social psychology, 47(6), 1191.

Calder, B. J., \& Lutz, R. J. (1972). An investigation of some alternatives to the linear attitude model. ACR Special Volumes.

Chiu, C. J., Hu, Y. H., Lin, D. C., Chang, F. Y., Chang, C. S., \& Lai, C. F. (2016). The attitudes, impact, and learning needs of older adults using apps on touchscreen mobile devices: Results from a pilot study. Computers in Human Behavior, 63, 189-197.

Eagly, A. H., \& Chaiken, S. (1993). The psychology of attitudes. Harcourt Brace Jovanovich College Publishers.

Eagly, A. H., \& Chaiken, S. (2007). The advantages of an inclusive definition of attitude. Social cognition, 25(5), 582-602.

Findlater, L., Moffatt, K., Froehlich, J. E., Malu, M., \& Zhang, J. (2017, May). Comparing touchscreen and mouse input performance by people with and without upper body motor impairments. In Proceedings of the 2017 CHI Conference on Human Factors in Computing Systems (pp. 6056-6061). ACM.

Fishbein, M. (1963). An investigation of the relationships between beliefs about an object and the attitude toward that object. Human relations, 16(3), 233-239.

Gorce, P., Nadine, V., \& Motti, L. (2017). Interaction techniques for older adults using touchscreen devices: a literature review from 2000 to 2013. Journal d'Interaction Personne-Système, 3.

Jain, V. (2014). 3D model of attitude. International Journal of Advanced Research in Management and Social Sciences, 3(3), 1-12.

Kraus, L. (2017). Disability Statistics Annual Report. Durham, NH: University of New Hampshire.

Page, T. (2017). Touchscreen and perceived usability: A comparison of attitudes between older and younger mobile device users. i-manager's Journal on Mobile Applications and Technologies, 3(1), 1-16.

Schipor, M. D., \& Vatavu, R. D. (2017, June). Coping strategies of people with low vision for touch input: A lead-in study. In E-Health and Bioengineering Conference (EHB), 2017 (pp. 357-360). IEEE. 
Schipor, O. A., Pentiuc, S. G., \& Schipor, M. D. (2011). The Utilization of Feedback and Emotion Recognition in Computer based Speech Therapy System. Elektronika ir Elektrotechnika, 109(3), 101-104.

Schipor, O. A., \& Schipor, M. D. (2007). Computer Assisted Therapy of Dyslalia. The Knowledge Based Organization, 13, 22-25.

Schwarz, N., \& Bohner, G. (2001). The construction of attitudes. Blackwell handbook of social psychology: Intraindividual processes, 1, 436-457.

Schwarzer, R. A. L. F., \& Luszczynska, A. (2005). Social cognitive theory. Predicting bealth behaviour, 2, 127-169.

Tai, K., Blain, S., \& Chau, T. (2008). A review of emerging access technologies for individuals with severe motor impairments. Assistive Technology, 20(4), 204221.

Vatavu, R. D., Cramariuc, G., \& Schipor, D. M. (2015). Touch interaction for children aged 3 to 6 years: Experimental findings and relationship to motor skills. International Journal of Human-Computer Studies, 74, 54-76.

WHO, W. (2011). World report on disability. Geneva: WHO. 